\title{
Contralateral tinnitus and hearing loss due to a tumor at the region of inferior colliculus: illustrative case
}

\author{
Kourosh Eftekharian, MD, MPH, ${ }^{1}$ Guive Sharifi, MD, ${ }^{2}$ Ali Eftekharian, $M D,{ }^{1}$ and Farahnaz Bidari-Zerehpoosh, $M D^{3}$ \\ Departments of ${ }^{1}$ Otolaryngology, ${ }^{2}$ Neurosurgery, and ${ }^{3}$ Pathology, Loghman Hospital, Shahid Beheshti University of Medical Sciences, Tehran, Iran
}

BACKGROUND Subjective hearing loss resulting from unilateral lesions of the quadrigeminal cistern region or inferior colliculus is a rare entity.

OBSERVATIONS The authors presented the case of a 32-year-old man with a history of 4 months of nonpulsatile tinnitus and 1 month of unilateral sensorineural hearing loss. He was otherwise healthy with no significant previous medical history. Intravenous gadolinium contrast magnetic resonance imaging of the head revealed a lesion at the region of the left inferior colliculus suggestive of a lipoma. The tumor was removed, and the patient had no significant hearing change afterward, with an improvement in tinnitus. The histological examination demonstrated the tumor as a fibrolipoma.

LESSONS Contralateral subjective hearing loss is an uncommon presentation for a lesion in the region of the inferior colliculus.

https://thejns.org/doi/abs/10.3171/CASE21624

KEYWORDS tinnitus; hearing loss; inferior colliculi; lipoma; brain neoplasms

Astrocytoma is the most common tumor of the quadrigeminal plate, whereas other tumors have been described more rarely. ${ }^{1}$

Lipomas make up only $0.1-0.5 \%$ of intracranial lesions. They tend to be placed at the midline, most commonly in the pericallosal cistern. The quadrigeminal cistern is the second most common location for intracranial lipomas. ${ }^{2}$

Because of the central auditory system's extensive interconnected pathways, subjective hearing loss resulting from unilateral lesions of the quadrigeminal cistern region or inferior colliculus is rare.

Here we discuss a case of quadrigeminal plate lipoma with uncommon presentations.

\section{Illustrative Case}

\section{History and Examination}

A 32-year-old right-handed man with a 4-month history of tinnitus in the right ear presented to our clinic. The tinnitus was continuous nonpulsatile, and he could not name any triggering factor. $\mathrm{He}$ also reported unilateral right ear hearing loss from 1 month before he arrived at our clinic. The patient had no symptoms, such as vertigo or headache, other than hearing loss and tinnitus.
He complained of difficulty hearing telephone calls with the right ear and trouble understanding speech in noisy environments. The patient had no previous significant medical problems, including hearing problems in his medical history or his family's medical history. As the first step, pure tone audiometry was performed (Fig. 1A), which revealed a sloping severe sensorineural hearing loss in the right ear at frequencies higher than $500 \mathrm{~Hz}$.

Afterward, head magnetic resonance imaging (MRI) with and without intravenous gadolinium administration showed a $20-\mathrm{mm}$ lesion centered in the left quadrigeminal cistern with minimal mass effect over the region of the left inferior colliculus. The MRI demonstrated high signal intensity on both T1 and T2 sequences (Fig. 2), with a further uniform signal drop in fat, saturated postcontrast T1 and T2 images in favor of lipoma. No related abnormality was identified in magnetic resonance angiography and magnetic resonance venography images.

\section{Operation and Postoperative Course}

Using this imaging evidence, the patient was electively prepared for removal of the brain neoplasm. The patient received a midline suboccipital craniotomy in the semiseated position. The tumor was 

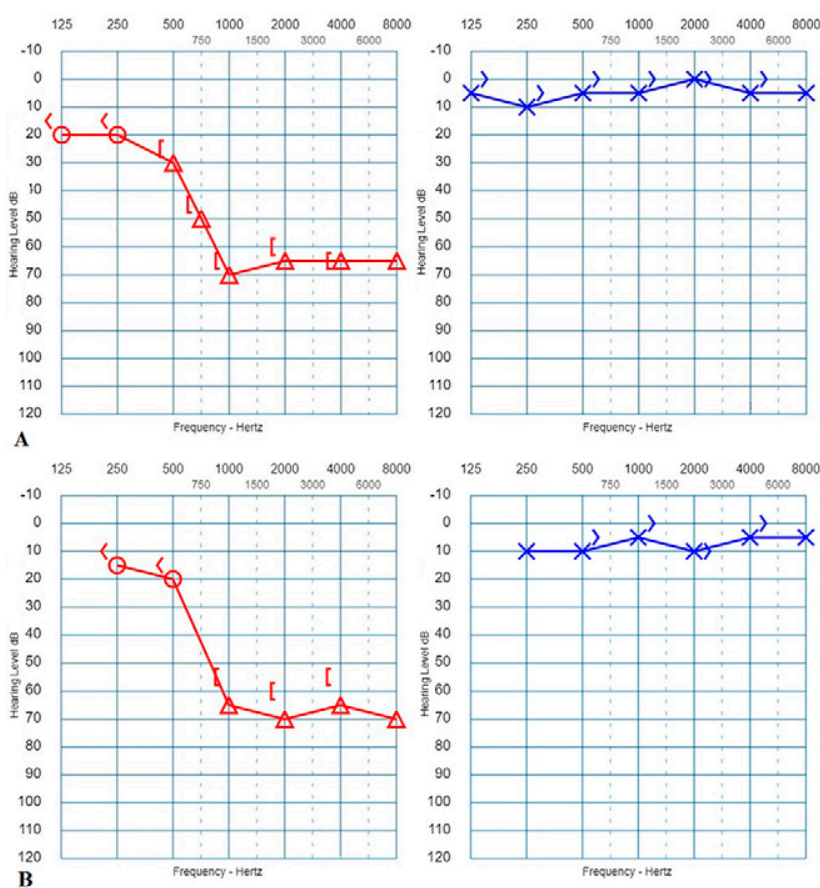

FIG. 1. A: Patient's audiogram before surgery. Right ear speech discrimination score $(S D S)=80$; left ear $S D S=100$. B: Patient's audiogram after surgery. Right ear $S D S=80$; left ear $S D S=100$.

resected via a supracerebellar infratentorial approach with bedside monitoring. Intraoperatively, the tumor presented as a white-yellowish mass. A patch of arachnoid covered the lesion, and a thick adhesion was present between the superior vermis and colliculus. After releasing the adhesion and opening the thick capsule-like barrier, we encountered the mass. The origin of the lipoma seemed to be within the colliculus itself. However, exophytic dorsal growth had placed the tumor in the quadrigeminal cistern. The tumor was removed entirely.

After surgery, the patient's course was uneventful, and he was discharged 2 days after the operation. The audiometry test after the surgery showed no further exacerbations in the patient's hearing, which concurred with the patient's subjective hearing perception (Fig. 1B). In addition, although not wholly resolved, the patient's tinnitus significantly improved, with no further hearing loss after the operation.

\section{Histological Examination}

Histopathologic examination exhibited lobulated mature adipose tissue without any atypia, necrosis, or lipoblast. In some sections, fibrous areas and few scattered vascular structures attached to glial
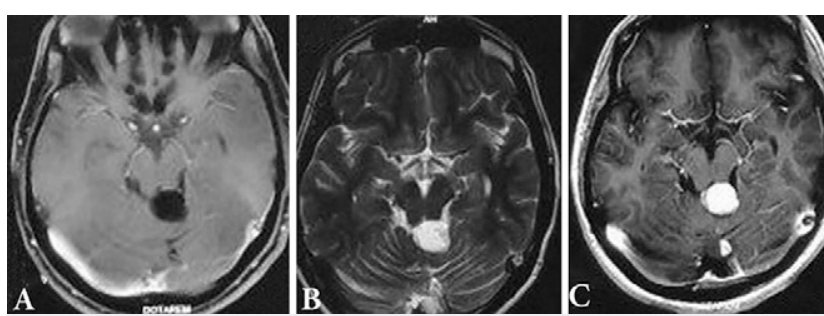

FIG. 2. Brain tumor. Axial T1-weighted (A), T2-weighted (B), and T1weighted postgadolinium (C) MRI.

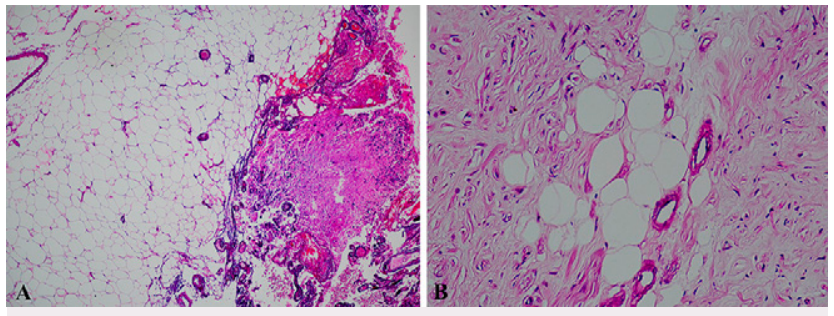

FIG. 3. Pathological examination. A: Lipoma composed of mature adipocytes with few vessels at the left side and a small part of glial tissue attached to it at the right side (hematoxylin and eosin stain, low power field). Original magnification $\times 100$. B: Fibrous proliferation, some remaining adipocytes embedded within it (hematoxylin and eosin stain, high power field). Original magnification $\times 400$.

tissue were seen. Based on these histopathologic findings, the final diagnosis was fibrolipoma (Fig. 3).

\section{Discussion}

\section{Observations}

Lipomas are rare intracranial lesions. They are usually at the midline, most commonly in the pericallosal cistern, and their second most common location is the quadrigeminal cistern. ${ }^{2}$

Intracranial lipomas result from the maldevelopment of embryonic primitive meninx rather than neoplastic or hamartomatous origin. ${ }^{2}$

The inferior colliculus is located in the midbrain, just caudal to the superior colliculus. From the cochlear nucleus, three fiber tracts project auditory information to the contralateral inferior colliculus. These fiber tracts collectively form the lateral lemniscus. Some fibers from the cochlear nuclei do not cross the midline and instead project into the ipsilateral inferior colliculus. Connections also exist between the cochlear nuclei on both sides; they represent the most peripheral connections between the auditory pathways bilaterally although these connections' function is not well understood. ${ }^{3}$

The inferior colliculus processes the information it receives and sends fibers to the thalamus's medial geniculate body. Fibers that project from the inferior colliculus to the medial geniculate body number $\sim 250,000$, which is almost ten times as many as auditory nerves. This increase in the number of nerve fibers at the inferior colliculus indicates the substantial amount of signal processing in the central auditory system. ${ }^{3}$

Most tumors of the quadrigeminal plate have a silent clinical course. They often become symptomatic by obliterating the aqueduct, causing hydrocephalus. ${ }^{1}$

Dysfunction below or within the cochlear nuclei produces ipsilateral auditory abnormalities, whereas disorders rostral to the cochlear nuclei may result in bilateral abnormalities. The multiplicity of parallel and overlapping auditory pathways and the extensive connections of the auditory brainstem nuclei are responsible for binaural interaction and ensure redundancy in the system. This redundancy may explain why small brainstem lesions are often clinically silent. ${ }^{4}$

Literature's reported auditory findings of the unilateral lesions in the region of inferior colliculus are bilateral normal pure tone audiogram, tinnitus at the contralateral side, normal as well as contralateral or bilaterally impaired speech discrimination and detection threshold tests, a deficit in sound localization contralateral to the lesion, difficulty in 
understanding speech in noisy environments, and bilateral abnormality in auditory brainstem response. ${ }^{5-7}$

Our patient had contralateral tinnitus, similar to other cases in the literature. However, it showed sloping low to high tone hearing loss contralateral to the lesion in pure tone audiogram. To our knowledge, there has been no similar case in the literature of this type of hearing loss due to a unilateral lesion of the inferior colliculus region.

Some fibers from the cochlear nuclei that do not cross the midline and project into the ipsilateral inferior colliculus instead as well as connections that also exist among the cochlear nuclei and colliculi on both sides explain why pure tone audiograms of the reported cases in the literature showed normal results. They also explain why some of the cases had normal speech discrimination and detection threshold. However, it is not clear why our case involved an abnormal pure tone audiogram result.

For unilateral lesions in the inferior colliculus region, tinnitus is only reported to be contralateral to the lesion. ${ }^{6,8}$ If we suppose that connections among the cochlear nuclei and the inferior colliculi can explain the normality of pure tone audiogram, then why do we have tinnitus only on the contralateral side of the lesion?

\section{Lessons}

There seem to be other mechanisms involved in the central auditory pathway that are not yet completely understood. A better understanding of these pathways may help us determine why the tinnitus is only contralateral to inferior colliculus's unilateral lesions and why our patient had a contralateral pure tone impairment. Thus, to explain our case, we need more thorough investigation on the auditory pathways from cochlear nuclei to inferior colliculi.

\section{References}

1. Montibeller GR, Stan AC, Krauss JK, Nakamura M. Calcifying pseudoneoplasm of the inferior colliculus: an unusual location for a rare tumor: case report. Neurosurgery. 2009;65(5):E1005-E1006.
2. Yilmaz MB, Egemen E, Tekiner A. Lipoma of the quadrigeminal cistern: report of 12 cases with clinical and radiological features. Turk Neurosurg. 2015;25(1):16-20.

3. Chien WW, Lee DJ. Physiology of the auditory system. In: Flint PW, Haughey BH, Lund VJ, et al., eds. Cummings Otolaryngology: Head and Neck Surgery. 7th ed. Elsevier; 2020:1945-1957.

4. Celesia GG. Hearing disorders in brainstem lesions. Handb Clin Neurol. 2015;129:509-536.

5. Champoux F, Paiement P, Mercier C, Lepore F, Lassonde M, Gagné JP. Auditory processing in a patient with a unilateral lesion of the inferior colliculus. Eur J Neurosci. 2007;25(1):291-297.

6. Kwee IL, Matsuzawa H, Nakada K, Fuji Y, Nakada T. Inferior colliculus syndrome: clinical magnetic resonance microscopy anatomic analysis on a 7 T system. SAGE Open Med Case Rep. 2017;5:2050313X17745209.

7. Litovsky RY, Fligor BJ, Tramo MJ. Functional role of the human inferior colliculus in binaural hearing. Hear Res. 2002;165(1-2):177-188.

8. Stimmer H, Borrmann A, Löer C, Arnold W, Rummeny EJ. Monaural tinnitus from a contralateral inferior colliculus hemorrhage. Audiol Neurotol. 2009;14(1):35-38.

\section{Disclosures}

The authors report no conflict of interest concerning the materials or methods used in this study or the findings specified in this paper.

\section{Author Contributions}

Conception and design: K Eftekharian, Sharifi, A Eftekharian. Acquisition of data: Sharifi, Bidari-Zerehpoosh. Analysis and interpretation of data: K Eftekharian. Drafting the article: K Eftekharian, Sharifi. Critically revising the article: K Eftekharian, Sharifi. Reviewed submitted version of manuscript: K Eftekharian, Sharifi. Approved the final version of the manuscript on behalf of all authors: K Eftekharian. Administrative/technical/material support: Sharifi. Study supervision: Sharifi, A Eftekharian.

\section{Correspondence}

Kourosh Eftekharian: Loghman Hospital, Shahid Beheshti University of Medical Sciences, Tehran, Iran. kourosheftkharian@gmail.com. 